\title{
DENJOY AND P-PATH INTEGRALS ON COMPACT GROUPS IN AN INVERSION FORMULA FOR MULTIPLICATIVE TRANSFORMS
}

\author{
Francesco Tulone
}

\begin{abstract}
Denjoy and $\mathcal{P}$-path Kurzweil-Henstock type integrals are defined on compact subsets of some locally compact zero-dimensional abelian groups. Those integrals are applied to obtain an inversion formula for the multiplicative integral transform.
\end{abstract}

\section{Introduction}

In [8, an inversion formula for the multiplicative integral transform was obtained for the case of any locally compact zero-dimensional abelian periodic group. This result does not cover the case of transforms convergent to Denjoy-Khintchine integrable functions because this integral is incompatible with $\mathcal{P}$-adic integral used in the above result (see [2]).

To overcome this difficulty, we consider here a little bit less general class of zero-dimensional groups and another Kurzweil-Henstock type integral on them. This gives us an opportunity to get an inversion formula for the multiplicative integral transform convergent to a Denjoy-Khintchine integrable function in the case of a class of groups considered here.

This problem is a generalization of that of recovering the coefficients of a convergent series with respect to characters of a compact zero dimensional abelian group which was considered in [7]. A similar problem related to some special groups was considered in [6].

2000 Mathematics Subject Classification: 43A70, 43A25, 26A39, 42C10.

Keywords: locally compact zero-dimensional abelian group, characters of a group, Denjoy integral, Kurzweil-Henstock $\mathcal{P}$-path integral, multiplicative integral transform, inversion formula. 


\section{FRANCESCO TULONE}

\section{Preliminaries}

Let $G$ be a zero-dimensional locally compact abelian periodic group which satisfies the second countability axiom. We can introduce (see [1]) a topology in such a group using a chain of subgroups

$$
\ldots \supset G_{-n} \supset \ldots \supset G_{-2} \supset G_{-1} \supset G_{0} \supset G_{1} \supset G_{2} \supset \ldots \supset G_{n} \supset \ldots
$$

with $G=\cup_{n=-\infty}^{+\infty} G_{n}$ and $\{0\}=\cap_{n=-\infty}^{+\infty} G_{n}$. The subgroups $G_{n}$ are clopen sets with respect to this topology. As $G$ is periodic, the factor group $G_{n} / G_{n+1}$ is finite for each $n$ and this implies that $G_{n}$ is compact. Let $p_{n+1}$ be an order of $G_{n} / G_{n+1}$ if $n \geq 0$ and $p_{-n}$ an order of $G_{-n} / G_{-n+1}$ if $n \geq 1$. We can suppose that all $p_{n}$ are prime numbers. So, the group $G$ defines a sequence

$$
\mathcal{P}=\left\{p_{j}\right\}_{j=-\infty}^{+\infty}, \quad j \neq 0 .
$$

We also consider the reverse sequence

$$
\mathcal{P}^{\prime}=\left\{p_{j}^{\prime}\right\}_{j=-\infty}^{+\infty}, \quad j \neq 0,
$$

where $p_{j}^{\prime}=p_{-j}$ for $j \in \mathbb{Z} \backslash\{0\}$. Moreover, we set $m_{0}=1, m_{j}=\prod_{s=1}^{j} p_{s}$, $m_{-j}=\prod_{s=-1}^{-j} p_{s}$.

We remind that a complex function $\chi$ on a locally compact abelian group $G$ is called a character of $G$ if $|\chi(g)|=1$ for all $x \in G$ and if a functional equation

$$
\chi\left(g_{1}+g_{2}\right)=\chi\left(g_{1}\right)+\chi\left(g_{2}\right)
$$

is satisfied for all $g_{1}, g_{2} \in G$. The set of all continuous characters of $G$ forms a group $X$, the dual group of $G$, if the addition is defined by

$$
\left(\chi_{1}+\chi_{2}\right)(g)=\chi_{1}(g) \cdot \chi_{2}(g),
$$

where $x \in G$ and $\chi_{1}, \chi_{2} \in X$. In view of the duality between $G$ and $X$, see ([10]), it is customary to write $(g, \chi)$ in place of $\chi(g)$.

In what follows, $X$ will denote the dual group of the group $G$ described above. It is known (see [1]) that under the assumption imposed on $G$ the group $X$ is also a periodic locally compact zero-dimensional abelian group and we can represent it to be a sum of increasing sequence of subgroups:

$$
\ldots \supset X_{n} \supset \ldots \supset X_{2} \supset X_{1} \supset X_{0} \supset X_{-1} \supset X_{-2} \supset \ldots \supset X_{-n} \supset \ldots
$$

introducing a topology in $X$. Then, $X=\cup_{i=-\infty}^{+\infty} X_{i}$ and $\cap_{i=-\infty}^{+\infty} X_{i}=\left\{\chi^{(0)}\right\}$ where $\left(g, \chi^{(0)}\right)=1$ for all $g \in G$. For each $n \in \mathbb{Z}$, the group $X_{n}$ is the annihilator of $G_{n}$, i.e.,

$$
X_{n}=G_{n}^{\perp}=\left\{\chi \in X:(g, \chi)=1 \quad \text { for all } g \in G_{n}\right\} .
$$

The factor groups $X_{n+1} / X_{n}=G_{n+1}^{\perp} / G_{n}^{\perp}$ and $G_{n} / G_{n+1}$ are isomorphic (see [1]). So, the order of $X_{n+1} / X_{n}$ is $p_{n+1}$ if $n \geq 0$ and $p_{n}$ if $n \leq-1$. 


\section{DENJOY AND P-PATH INTEGRALS ON COMPACT GROUPS}

It can be shown (see [8]) that any element $g \in G$ can be represented in the form

$$
g=[g]+\{g\}
$$

and any element $\chi \in X$ in the form

$$
\chi=[\chi] \cdot\{\chi\}
$$

in such a way that the following properties hold:

1) $\{g\} \in G_{0}$ and $\{\chi\} \in X_{0}=G_{0}^{\perp}$. So, $(\{g\},\{\chi\})=1$, and

$$
(g, \chi)=(\{g\},[\chi]) \cdot([g],[\chi]) \cdot([g],\{\chi\}) .
$$

2) $[\chi] \in G_{m}^{\perp}$ for some $m=m(\chi) \in \mathbb{N}$ and $[\chi] \backslash_{G_{0}}$ is a character of the subgroup $G_{0}$.

3) $([g],[\chi])$ is constant if $g$ belongs to a fixed coset of $G_{0}$ and $\chi$ belongs to a fixed coset of $X_{0}$.

Using the duality between $G$ and $X$, we can state that $g$ represents a character of $X$ and similarly to property 2$),[g] \backslash_{X_{0}}$ is a character of $X_{0}$. So, $([g],\{\chi\})$ is a value of this character at the point $\{\chi\}$.

Therefore, according to (6), if $g$ belongs to a fixed coset of $G_{0}$ and $\chi$ belongs to a fixed coset of $X_{0}$, we can represent $(g, \chi)$, up to a constant $([g],[\chi])$, as a product of $(\{g\},[\chi])$ considered as a value of the character $[\chi]$ at $\{g\}$, and $([g],\{\chi\})$ considered as a value of the character $[g]$ at $\{\chi\}$.

It is also shown (see [8]) that we can map the groups $G$ and $X$ to the interval $[0,+\infty)$ by mappings

$$
\varphi(g)=x=\{x\}+[x]
$$

and

$$
\psi(\chi)=x^{\prime}=\left\{x^{\prime}\right\}+\left[x^{\prime}\right]
$$

where $\{a\}$ is the fractional part of $a$ and $[a]$ the integer part of $a$. Moreover, the preimage of $[x]$, under the mapping $\varphi$, is $[g]$ and the one of $\{x\}$ is $\{g\}$. This explains the notation in (44). In the same way, $\psi([\chi])=\left[x^{\prime}\right]$ and $\psi(\{\chi\})=\left\{x^{\prime}\right\}$.

In this way, the subgroup $G_{n}, n \geq 0$, is mapped onto the interval $\left[0, \frac{1}{m_{n}}\right]$ and the respective cosets are mapped onto intervals $I_{n}^{k}=\left[\frac{k}{m_{n}}, \frac{k+1}{m_{n}}\right]$ with $k=0,1, \ldots$ We denote these cosets by $G_{n}^{k}$ with $G_{n}^{0}=G_{n}$. In particular, for $n=0$ we map $G_{0}$ onto $[0,1]$ and respective cosets onto intervals $[k, k+1]$. In case $n<0$, the group $G_{n}$ is mapped onto interval $\left[0, m_{n}\right]$ and respective cosets $G_{n}^{k}$ are mapped onto intervals $\left[\mathrm{km}_{n},(k+1) \mathrm{m}_{n}\right]$. We call all the above mentioned images of cosets the $\mathcal{P}$-adic intervals. If $n$ is fixed, we refer to $I_{n}^{k}$ as to intervals of a rank $n$. The set of all $\mathcal{P}$-adic intervals is denoted by $\mathcal{I}_{\mathcal{P}}$.

In a similar way, we can define $\mathcal{P}^{\prime}$-adic intervals associated with the group $X$ and the sequence $\mathcal{P}^{\prime}$ (see (2) ). They are images, under mapping (8), of the respective cosets of the subgroup $X_{n}$ denoted by $X_{n}^{s}$. 
Note that the mappings (77) and (8) are one-one correspondences between the interval $[0,+\infty)$ and the groups $G$ and $X$, respectively, up to a countable number of $\mathcal{P}$-adic $\left(\mathcal{P}^{\prime}\right.$-adic) rational points, i.e., points $\frac{t}{m_{k}}\left(\right.$ or $\left.\frac{t}{m_{-k}}\right)$ with $t, k=0,1,2, \ldots$ Such a point $x$ has two preimages corresponding to the finite and to the infinite expansion of $x$, respectively. We denote by $Q_{\mathcal{P}}$ the set of all $\mathcal{P}$-adic rational points and by $Q_{\mathcal{P}^{\prime}}$ the set of all $\mathcal{P}^{\prime}$-adic rational points. We agree to use only finite expansions for $\mathcal{P}$-adic (and $\mathcal{P}^{\prime}$-adic) rational points so that the inverse mappings $\varphi^{-1}$ and $\psi^{-1}$ make sense on $[0, \infty)$.

We consider the Haar measure $\mu_{G}$ on the group $G$ and we normalize it so that $\mu_{G}\left(G_{0}\right)=1$. Then the measure of any coset $G_{n}^{k}$ of $G_{n}$ coincides with the length of the $\mathcal{P}$-adic interval which is the image of $G_{n}^{k}$, i.e.,

$$
\mu_{G}\left(G_{n}^{k}\right)=\frac{1}{m_{n}} \quad \text { if } \quad n \geq 0 \quad \text { and } \quad \mu_{G}\left(G_{n}^{k}\right)=m_{n} \quad \text { if } \quad n<0 .
$$

In the same way, we introduce the measure $\mu_{X}$ on $X$ so that $\mu_{X}\left(X_{0}\right)=1$ and

$$
\mu_{X}\left(X_{n}^{s}\right)=\frac{1}{m_{n}} \quad \text { if } \quad n \leq 0 \quad \text { and } \quad \mu_{X}\left(X_{n}^{s}\right)=m_{n} \quad \text { if } \quad n>0 .
$$

Note that, under the above mentioned mappings $\varphi$ and $\psi$, the image of each set of Haar measure zero on the group is a set of Lebesgue measure zero on $[0,+\infty)$.

We denote by $\mathcal{R}_{G}$ the ring generated by the family of all cosets $G_{n}^{k}, n \in \mathbb{Z}$, $k=0,1,2, \ldots$, and by $\mathcal{R}_{\mathcal{P}}$ the ring generated by $\mathcal{I}_{\mathcal{P}}$. Note that for each $g \in G$ there exists a decreasing sequence $\left\{G_{n}^{k(n)}\right\}_{n}$ of cosets such that $g \in \cap_{n} G_{n}^{k(n)}$.

\section{P-path Kurzweil-Henstock integral and Denjoy-Khintchine integral on the group $G$}

Now, we introduce a Henstock-Kurzweil type integral with respect to the system of $\mathcal{P}$-paths and also Denjoy-Khintchine integral on compact subgroups of the considered group $G$ and $X$.

With each $\mathcal{P}$-adic irrational point $x \in[0, \infty)$, i.e., a point $x \in[0, \infty) \backslash Q_{\mathcal{P}}$, we associate a unique nested sequence

$$
\left\{\varphi\left(G_{n}^{k(n)}\right)\right\}_{n}=\left\{I_{n}^{k(n)}\right\}=\left\{\left[a_{n}(x), b_{n}(x)\right]\right\}_{n=0}^{\infty}
$$

of $\mathcal{P}$-adic intervals converging to $x$ so that

$$
\{x\}=\bigcap_{n=0}^{\infty}\left[a_{n}(x), b_{n}(x)\right] .
$$


If $x$ is a $\mathcal{P}$-adic rational point, then there exist two nested sequences (9) - the left one and the right one - for which $x$, starting with some $n$, is the common end-point.

Using the notation given by (9), we define for a $\mathcal{P}$-adic irrational point $x$, the sequences $\mathcal{P}_{x}^{-}=\left\{a_{n}(x)\right\}$ and $\mathcal{P}_{x}^{+}=\left\{b_{n}(x)\right\}$. If $x$ is a $\mathcal{P}$-adic rational point, we use the same notation $\mathcal{P}_{x}^{+}$and $\mathcal{P}_{x}^{-}$for sequences with $a_{n}(x)$ being the left end-point of interval of rank $n$ of the above mentioned left nested sequence associated with $x$, and $b_{n}(x)$ being the right end-point of interval of rank $n$ of the right nested sequence.

Definition 3.1. The set $\mathcal{P}_{x}=\mathcal{P}_{x}^{+} \bigcup \mathcal{P}_{x}^{-} \bigcup\{x\}$ is called the $\mathcal{P}$-path leading to $x$. If $E \in \mathcal{R}_{\mathcal{P}}$, the collection $\left\{\mathcal{P}_{x}: x \in E\right\}$ is called the system of $\mathcal{P}$-paths on $E$.

The continuity and the derivative at a point $x$ with respect to the set $\mathcal{P}_{x}$ are called $\mathcal{P}$-path continuity and $\mathcal{P}$-path derivative. In the same way, we define $\mathcal{P}$-path upper and lower derivatives.

Definition 3.2. Let $\delta$ be a positive function defined on $E \in \mathcal{R}_{\mathcal{P}}$. A collection of interval-point pairs $\left\{\left(\left[u_{j}, v_{j}\right], x_{j}\right)\right\}_{j=1}^{k}$ is called $\delta$-fine $\mathcal{P}$-partition of $E$ if the intervals $\left[u_{j}, v_{j}\right]$ are non-overlapping, $E=\cup_{j=1}^{k}\left[u_{j}, v_{j}\right]$ and for each $j$ we have $u_{j}, v_{j} \in \mathcal{P}_{x}, u_{j} \leq x_{j} \leq v_{j}$ and $\max \left\{v_{j}-x_{j}, x_{j}-u_{j}\right\}<\delta\left(x_{j}\right)$.

It is not difficult to check that for any $E \in \mathcal{R}_{\mathcal{P}}$, any system of $\mathcal{P}$-paths on $E$, and for any positive function $\delta$ defined on $E$ there exists a $\delta$-fine $\mathcal{P}$-partition of $E$.

Definition 3.3. A complex valued function $f$ on $E \in \mathcal{R}_{\mathcal{P}}$ is said to be Kurzweil-Henstock integrable with respect to the system of $\mathcal{P}$-paths or, in brief, $H_{\mathcal{P}}$-integrable on $E$, with integral value $A$, if for every $\varepsilon>0$, there exists a positive function $\delta$ on $E$ such that for any $\delta$-fine $\mathcal{P}$-partition of $E$ we have

$$
\left|\sum_{j=1}^{k} f\left(x_{j}\right)\left(v_{j}-u_{j}\right)-A\right|<\varepsilon .
$$

Then we write $\left(H_{\mathcal{P}}\right) \int_{E} f=A$.

It is easy to check that a function which is equal to zero almost everywhere on $E \in \mathcal{R}_{\mathcal{P}}$, is $H_{\mathcal{P}}$-integrable to zero on $E$. This justifies the following extension of Definition 3.3 to the case of functions defined only almost everywhere on $E$ (for short, a.e.).

Definition 3.4. A complex valued function $f$ defined a.e. on $E \in \mathcal{R}_{\mathcal{P}}$ is said to be $H_{\mathcal{P}}$-integrable on $E$ with integral value $A$ if the function

$$
f_{1}(x)= \begin{cases}f(x), & \text { where } f \text { is defined } \\ 0, & \text { otherwise }\end{cases}
$$


is $H_{\mathcal{P}}$-integrable on $E$ to $A$ in the sense of Definition 3.3 .

We can use this definition to introduce the respective $H_{G}$-integral on certain subsets of the considered group $G$.

Definition 3.5. Let $E \in \mathcal{R}_{\mathcal{P}}$ be the image of $T \in \mathcal{R}_{G}$ under the mapping $\varphi$ (see (7)). Then a complex-valued function $f$ defined almost everywhere on $T$ is $H_{G^{-}}$integrable on $T$, with the value $A$, if the function $F(x)=f\left(\varphi^{-1}(x)\right)$ is $H_{\mathcal{P}}$-integrable on $E$ with the value $A$ in the sense of Definition 3.4 , and we write $\left(H_{G}\right) \int_{T} f d \mu_{G}=A$.

Remark 3.1. We note that the above definition depends on the sequence $\mathcal{P}$ defined by the group $G$. So, if we consider a similar definition of the $H_{X}$-integral on a subset of the group $X$, then we should use a sequence $\mathcal{P}^{\prime}$ defined by $X$ and the respective $H_{\mathcal{P}^{\prime} \text {-integral. }}$

Now, we shall give a definition of Denjoy-Khintchine integral on compact subgroups of a locally compact group.

Definition 3.6. A real function $\Phi$ defined on $[a, b]$ is an ACG-function if $[a, b]=\cup_{i=1}^{\infty} B_{i}$ and $\Phi$ is absolutely continuous on each $B_{i}$.

Definition 3.7. A real function $f$ defined almost everywhere on $[a, b]$ is Denjoy-Khintchine integrable on $[a, b]$, briefly, D-integrable, if there exists an $A C G$ function $\Phi$ such that $\Phi_{a p}^{\prime}=f$ almost everywhere, where $\Phi_{a p}^{\prime}$ denotes the approximate derivative (see [4]). The value of this integral is defined as $(D) \int_{a}^{b} f=\Phi(b)-\Phi(a)$.

DEFinition 3.8. A real function $f$ defined almost everywhere on $E=\cup_{i=1}^{n}\left[\alpha_{i}, \beta_{i}\right]$ is $D$-integrable on $E$ if $f$ is $D$-integrable on each $\left[\alpha_{i}, \beta_{i}\right]$ and the value of integral is $(D) \int_{E} f=\sum_{i=1}^{n}(D) \int_{\alpha_{i}}^{\beta_{i}} f$. In the case of a complex-valued function $f$, it is $D$-integrable on $E$ if both $\operatorname{Re} f$ and $\operatorname{Im} f$ are $D$-integrable with integrals $A$ and $B$, respectively, and the $D$-integral of $f$ is $A+i B$.

Definition 3.9. Let $E \in \mathcal{R}_{\mathcal{P}}$ be the image of $T \in \mathcal{R}_{G}$ under the mapping $\varphi$ (see (7)). Then a complex-valued function $f$ defined almost everywhere on $T$ is D-integrable on $T$, with the value $A$, if the function $F(x)=f\left(\varphi^{-1}(x)\right)$ is $D$-integrable on $E$ with the value $A$ in the sense of Definition 3.8 , and we write (D) $\int_{T} f d \mu_{G}=A$.

Remark 3.2. It is easy to check out that the $D$-integral (in the sense of the above definition) is invariant under translation given by some "integer part" $[g]$ of some element $g \in G$.

We need the following theorem (see [9]).

TheOREM 3.1. Suppose that the sequence (1) is bounded and a function $f$ defined on $[a, b]$ is both $H_{\mathcal{P}}$-integrable and D-integrable on $[a, b]$. If $\Phi$ is the indefinite 
$H_{\mathcal{P}}$-integral of $f$ and the upper and lower $\mathcal{P}$-path derivatives of $\operatorname{Re} \Phi$ and $\operatorname{Im} \Phi$ are bounded at each point of $[a, b]$, except a countable set, then the values of $H_{\mathcal{P}-}$ and D-integrals of $f$ on $[a, b]$ coincide.

The next result is of great importance for our further consideration. It is a direct consequence of the above theorem, related to the real line setting, and of Definitions 3.5 and 3.9 .

Theorem 3.2. Suppose that the sequence (1) is bounded and $E \in \mathcal{R}_{\mathcal{P}}$ is the image of $T \in \mathcal{R}_{G}$ under the mapping $\varphi$ (see(17)). If a $H_{G \text {-integrable on }}$ $T \in R_{G}$ function $f$ and its indefinite $H_{G}$-integral $\Phi$ are such that $f\left(\varphi^{-1}(x)\right)$ and $\Phi\left(\varphi^{-1}(x)\right)$ satisfy the conditions of the previous theorem, then the values of $H_{G^{-}}$and D-integrals of $f$ on $T$ coincide.

\section{An inversion formula for integral transforms convergent to a $D$-integrable function}

Using the notations for the cosets of $X_{0}$ and $G_{0}$ introduced in Section 2, we denote $[\chi] \backslash_{G_{0}}$ by $h_{s}$ if $[\chi] \in X_{0}^{s}$. In the same way, if $[g] \in G_{0}^{k}$, we denote $[g] \backslash_{x_{0}}$ by $h_{k}^{\prime}$. It can be easily checked that the sequence $\left\{h_{s}\right\}_{s=0}^{\infty}$ includes all the characters of $G_{0}$ and $\left\{h_{k}^{\prime}\right\}_{k=0}^{\infty}$ includes all the characters of $X_{0}$. This sequence $\left\{h_{s}\right\}$ having been translated on $[0,1]$ using the mapping (7), forms an orthonormal system $\left\{\xi_{s}\right\}$ on $[0,1]$, where $\xi_{s}(x)=h_{s}\left(\varphi^{-1}(x)\right)$ (see [1]).

The following theorem is proved in [7].

Theorem 4.1. Suppose that the sequence (11) is bounded, the series $\sum a_{s} \xi_{s}$ with respect to the above system $\left\{\xi_{s}\right\}$ converges a.e. on $[0,1]$ to a function $f$ and everywhere on $[0,1] \backslash Q_{\mathcal{P}}$, we have

$$
\limsup _{n \rightarrow \infty}\left|S_{n}(x)\right|<+\infty
$$

where $S_{n}=\sum_{s=0}^{n-1} a_{s} \xi_{s}$. Then $f$ is $H_{\mathcal{P}}$-integrable on $[0,1]$ (in the sense of Definition 3.4 and $\sum a_{s} \xi_{s}$ is $H_{\mathcal{P}}$-Fourier series of $f$.

We can reformulate this result in terms of the system $\left\{h_{s}\right\}$ defined on a group.

TheOREM 4.2. Let $G_{0}$ be a compact abelian zero-dimensional group such that the sequence (11) is bounded and let $\left\{h_{s}\right\}_{s}$ be a system defined as above. If the series $\sum_{s=0}^{\infty} a_{s} h_{s}$ converges a.e. on $G_{0}$ to a function $f$ and everywhere on $G_{0} \backslash \varphi^{-1}\left(Q_{\mathcal{P}}\right)$ we have

$$
\limsup _{n \rightarrow \infty}\left|\sigma_{n}(g)\right|<+\infty,
$$

where $\sigma_{n}=\sum_{s=0}^{n-1} a_{s} h_{s}$, then $f$ is $H_{G_{0}}$-integrable on $G_{0}$ and $\sum a_{s} h_{s}$ is the Fourier series of $f$ in the sense of integral defined in Definition 3.5 . 


\section{FRANCESCO TULONE}

It is known (see [7, Lemma 3.2]) that the condition (10) implies the boundedness of upper and lower derivatives of $\operatorname{Re} \Phi$ and $\operatorname{Im} \Phi$, where $\Phi$ is an indefinite $H_{\mathcal{P}}$-integral of $f$.

Combining this with the last two theorems and with Theorem 3.2 , we get.

TheOREM 4.3. Let $G_{0}$ and the system $\left\{h_{s}\right\}_{s}$ be as in Theorem 4.2 If the series $\sum_{s=0}^{\infty} a_{s} h_{s}$ converges a.e. on $G_{0}$ to a D-integrable function $f$ and everywhere on $G_{0} \backslash \varphi^{-1}\left(Q_{\mathcal{P}}\right)$ the inequality (11) holds, then $\sum a_{s} h_{s}$ is the Denjoy-Fourier series of $f$ (in the sense of the integral defined in Definition 3.9.

This theorem is a generalization of a similar result related to some special case of zero-dimensional group (see [5, Theorem 2]).

We also need the following theorem, which can be established using the same arguments as in [8, Theorem 8]:

TheOREM 4.4. The partial sums $\sigma_{m_{n}}(f, g)$ of the $H_{G}$-Fourier series (with respect to the system of characters of $G_{0}$ ) of a $H_{G}$-integrable on $G_{0}$ function $f$ are convergent to $f$ almost everywhere on $G_{0}$.

A continuum analog of the series $\sum_{s=0}^{\infty} a_{s} h_{s}$ is the integral transform

$$
\int_{X} a(\chi)(g, \chi) \mathrm{d} \mu_{X}
$$

with appropriately defined improper integral on $X$. So, the next theorem, which gives an inversion formula for this transform, can be considered as a generalization of Theorem 4.3 .

Theorem 4.5. Assume that $G$ is a group described in Section 2 such that the sequence (1) is bounded, $X$ is its dual group. Let $a(\chi)$ be a locally $H_{X^{-}}$integrable function and

$$
\lim _{s \rightarrow \infty}\left(H_{X}\right) \int_{\cup_{i=0}^{s} X_{0}^{i}} a(\chi)(g, \chi) \mathrm{d} \mu_{X}=f(g)
$$

a.e. on $G$, where $f$ is a D-integrable function on each $T \in \mathcal{R}_{G}$. Moreover, let us have everywhere on $G \backslash \varphi^{-1}\left(Q_{\mathcal{P}}\right)$

$$
\limsup _{s \rightarrow \infty}\left|\left(H_{X}\right) \int_{\cup_{i=0}^{s} X_{0}^{i}} a(\chi)(g, \chi) \mathrm{d} \mu_{X}\right|<+\infty .
$$

Then, the function $a(x)$ can be recovered from $f$ by the following inversion formula:

$$
a(\chi)=\lim _{n \rightarrow \infty}(D) \int_{G_{-n}} f(g) \overline{(g, \chi)} \mathrm{d} \mu_{G} \quad \text { a.e. on } \quad X .
$$


Pr o of. Suppose that $g \in G_{0}^{k}$. Then, for each $s=1,2, \ldots$, we have, according to (6),

$$
\begin{aligned}
& \left(H_{X}\right) \int_{\cup_{i=0}^{s} X_{0}^{i}} a(\chi)(g, \chi) \mathrm{d} \mu_{X} \\
& =\left(H_{X}\right) \int_{\cup_{i=0}^{s} X_{0}^{i}} a(\chi)(\{g\},[\chi]) \cdot([g],[\chi]) \cdot([g],\{\chi\}) \mathrm{d} \mu_{X} \\
& =\sum_{i=0}^{s}\left(H_{X}\right) \int_{X_{0}^{i}} a(\chi)(\{g\},[\chi]) \cdot([g],[\chi]) \cdot([g],\{\chi\}) \mathrm{d} \mu_{X} \\
& =\sum_{i=0}^{s} h_{i}(\{g\})\left(H_{X}\right) \int_{X_{0}^{i}} a(\chi)([g],[\chi]) h_{k}^{\prime}(\{\chi\}) \mathrm{d} \mu_{X} .
\end{aligned}
$$

Now, for any $g \in G_{0}^{k}$ for which the limit (12) exists, we get:

$$
f(g)=\lim _{s \rightarrow \infty} \sum_{i=0}^{s} h_{i}(\{g\})\left(H_{X}\right) \int_{X_{0}^{i}} a(\chi)([g],[\chi]) h_{k}^{\prime}(\{\chi\}) \mathrm{d} \mu_{X} .
$$

So, for such $g \in G_{0}^{k}$, the function $f(g)$ is the sum of series with respect to the system $\left\{h_{i}\right\}$ with coefficients

$$
b_{i}=\left(H_{X}\right) \int_{X_{0}^{i}} a(\chi)([g],[\chi]) h_{k}^{\prime}(\{\chi\}) \mathrm{d} \mu_{X}
$$

and this series is convergent almost everywhere on $G_{0}^{k}$.

In the same way, (13) and (15) imply, for any $g \in G_{0}^{k} \backslash \varphi^{-1}\left(Q_{\mathcal{P}}\right)$

$$
\limsup _{s \rightarrow \infty}\left|\sum_{i=0}^{s} h_{i}(\{g\})\left(H_{X}\right) \int_{X_{0}^{i}} a(\chi)([g],[\chi]) h_{k}^{\prime}(\{\chi\}) \mathrm{d} \mu_{X}\right|<\infty .
$$

Then, by Theorem 4.3, the coefficients $b_{i}$ are the Denjoy-Fourier coefficients of the $D$-integrable function $p(t)=f([g]+t)$ on $G_{0}$, i.e.,

$$
\begin{aligned}
b_{i}=\left(H_{X}\right) \int_{X_{0}^{i}} a(\chi)([g],[\chi]) h_{k}^{\prime}(\{\chi\}) \mathrm{d} \mu_{X} & =(D) \int_{G_{0}} p(t) \overline{h_{i}(t)} \mathrm{d} \mu_{G} \\
& =(D) \int_{G_{0}^{k}} f(g) \overline{h_{i}(\{g\})} \mathrm{d} \mu_{G}
\end{aligned}
$$


(the last equality is justified by Remark 3.2). By property 3) of Section 2, $([g],[\chi])$ is constant when $g \in G_{0}^{k}$ and $\chi \in X_{0}^{i}$. Hence, (17) implies

$$
\left(H_{X}\right) \int_{X_{0}^{i}} a(\chi) h_{k}^{\prime}(\{\chi\}) \mathrm{d} \mu_{X}=(D) \int_{G_{0}^{k}} f(g) \overline{([g],[\chi]) h_{i}(\{g\})} \mathrm{d} \mu_{G} .
$$

The rest of the proof follows the lines of the proof of Theorem 9 of $[8]$.

For a fixed $i$, the value

$$
\left(H_{X}\right) \int_{X_{0}^{i}} a(\chi) h_{k}^{\prime}(\{\chi\}) \mathrm{d} \mu_{X}
$$

is the Fourier coefficient in the system $\left\{\overline{h_{k}^{\prime}}\right\}$ of the $H_{X}$-integrable function $a(\chi)=$ $a([\chi]+\{\chi\})$ considered as a function of $\{\chi\} \in X_{0}$. Therefore, Theorem 4.4 being applied to the above system and the appropriate partial sums, implies

$$
\sum_{k=0}^{m_{-n}}\left(H_{X}\right) \int_{X_{0}^{i}} a(\chi) h_{k}^{\prime}(\{\chi\}) \mathrm{d} \mu_{X} \cdot \overline{h_{k}^{\prime}(\{\chi\})} \rightarrow a(\chi) \quad \text { a. e. on } X_{0}^{i} .
$$

Then, using (18) and (66), we get

$$
\begin{aligned}
& \lim _{n \rightarrow \infty} \sum_{k=0}^{m_{-n}}\left(H_{X}\right) \int_{X_{0}^{i}} a(\chi) h_{k}^{\prime}(\{\chi\}) \mathrm{d} \mu_{X} \cdot \overline{h_{k}^{\prime}(\{\chi\})} \\
& =\lim _{n \rightarrow \infty} \sum_{k=0}^{m_{-n}}(D) \int f(g) \overline{([g],[\chi]) h_{i}(\{g\})} \mathrm{d} \mu_{G} \cdot \overline{h_{k}^{\prime}(\{\chi\})} \\
& =\lim _{n \rightarrow \infty} \sum_{k=0}^{m_{-n}}(D) \int_{G_{0}^{k}} f(g) \overline{h_{i}(\{g\}) h_{k}^{\prime}(\{\chi\})([g],[\chi])} \mathrm{d} \mu_{G} \\
& =\lim _{n \rightarrow \infty}(D) \int_{\cup_{k=0}^{m}} f(g) \overline{(\{g\},[\chi])([g],\{\chi\})([g],[\chi])} \mathrm{d} \mu_{G} \\
& =\lim _{n \rightarrow \infty}(D) \int_{G_{-n}} f(g) \overline{(g, \chi)} \mathrm{d} \mu_{G}=a(\chi) \quad \text { a. e. on } \quad X_{0}^{i} .
\end{aligned}
$$

The last equality is true for any $i$. Therefore, we can write

$$
\lim _{n \rightarrow \infty}(D) \int_{G_{-n}} f(g) \overline{(g, \chi)} \mathrm{d} \mu_{G}=a(\chi) \quad \text { a. e. on } X .
$$

This completes the proof. 


\section{DENJOY AND $\mathcal{P}$-PATH INTEGRALS ON COMPACT GROUPS}

Remark 4.1. As both $D$-integral and $H_{X}$-integral obviously include the Lebesgue integral, the last theorem can be formulated, in particular, for functions $a$ and $f$ being locally Lebesgue integrable on the groups $X$ and $G$, respectively.

\section{REFERENCES}

[1] AGAEV, G. N.-VILENKIN, N. YA.-DZHAFARLI, G. M.-RUBISTEIN, A. I.: Multiplicative System of Functions and Harmonic Analysis on Zero-Dimensional Groups. "Ehlm", Baku, 1981. (In Russian)

[2] BONGIORNO, B.-DI PIAZZA, L.-SKVORTSOV, V. A.: The Ward property for a $\mathcal{P}$-adic basis and the $\mathcal{P}$-adic integral, J. Math. Anal. Appl. 285 (2003) 578-592.

[3] GOLUBOV, B.-EFIMOV, A.-SKVORTSOV, V.: Walsh Series and Transforms. Theory and Applications. Math. Appl. (Soviet Ser.), Vol. 64, Kluwer Academic Publishers, Dordrecht, 1991.

[4] GORDON, R. A.: The Integrals of Lebesgue, Denjoy, Perron, and Henstock Theory, Grad. Stud. Math., Vol. 4, Amer. Math. Soc., Providence, RI, 1994.

[5] SKVORTSOV, V. A.-KOROLEVA, M. P.: Series in multiplicative systems convergent to Denjoy-integrable functions, Sb. Math. 186 (1995), No. 12, 1821-1842; translation from Mat. Sb. 186 (1995), No. 12, 129-150.

[6] SKVORTSOV, V. A.-TULONE, F.: The P-adic Henstock integral in the problem of function representations by multiplicative transforms, Vestnik Moskov. Univ. Ser. I Mat. Mekh. 2005 (2005), No. 3, 41-44, translation in Moscow Univ. Math. Bull. 60 (2005), No. 3, 19-22.

[7] SKVORTSOV, V. A.-TULONE, F.: On the problem of recovering the coefficients of series with respect to characters of zero-dimensional groups, Tatra Mt. Math. Publ. 34 (2006), 307-320.

[8] SKVORTSOV, V. A.-TULONE, F.: Henstock type integral in harmonic analysis on zero-dimensional groups, J. Math. Anal. Appl. 322 (2006), 621-628.

[9] SKVORTSOV, V. A.-TULONE, F.: Integration of both the derivatives with respect to $\mathcal{P}$-paths and approximative derivatives, Mat. Zametki 85 (2009), No. 2, 283-291, translation in Math. Notes 85 (2009), 260-266.

[10] RUDIN, W.: Fourier Analysis on Groups. John Wiley \& Sons, New York, 1967.

Received September 4, 2007

Department of Mathematics
University of Palermo
via Archirafi 34
I-90123 Palermo
ITALY
E-mail: tulone@math.unipa.it 